\title{
APPlications OF NANOCRYSTAlS In DRUg DELIVERY: AN OVERVIEW
}

\author{
Sunita Dahiya* \\ Department of Pharmaceutical Sciences, School of Pharmacy, University of Puerto Rico, Medical Sciences \\ Campus, San Juan, PR 00936-5067, United States \\ ${ }^{*} E$-mail: sunita.dahiya@upr.edu \\ Tel.: +1 7877582525 (ext. 5413).
}

Received: July 22, 2018 / Revised: Aug 28, 2018 / Accepted: Aug 29, 2018

\begin{abstract}
Poor aqueous solubility has always been remained a challenge for delivery of drugs and show dissolution rate limited absorption. This problem seeks more attention in current time where number of poorly soluble candidates have increased up to 70 percent or more. Although extensive research efforts led to many successful marketed products using some commonly used solubility enhancement techniques such as micronization, salt formation, solid dispersion and complexation etc., each of the technique is associated with its own limitations. Nanonization approach involves transfer of poorly soluble materials into the nanodimension that changes their physical properties, and was utilized in pharmaceutical development of an innovative technology; known as drug nanocrystals. Among various nanotechnological approaches, nanocrystals have been emerged as one of the potential technology to overcome issues related to poor water solubility of drugs and its in vivo consequences. This article overviews the feasibility of nanocrystals for administration of poorly soluble drugs by oral, parenteral, dermal, mucosal, pulmonary, ocular and targeted delivery.
\end{abstract}

Key words: Poor solubility, Nanotechnology, Nanocrystal, Nanonization, Dissolution, Drug Delivery.

\section{INTRODUCTION}

Together with the permeability, the solubility behavior of a drug is a key determinant of its oral bioavailability. There have always been certain drugs for which solubility has presented a challenge to the development of a suitable formulation for oral administration. Examples such as griseofulvin, digoxin, phenytoin, sulphathiazole and chloramphenicol come immediately to mind. With the recent advent of high throughput screening of potential therapeutic agents, the number of poorly soluble drug candidates has risen sharply and the formulation of poorly soluble compounds for oral delivery now presents one of the most frequent and greatest challenges to formulation scientists in the pharmaceutical industry. Consideration of the modified equation given by Noyes and Whitney (Noyes and Whitney, 1897) provides some hints as to how the dissolution rate of even very poorly soluble compounds might be improved to minimize the limitations to oral availability. Table 1 broadly summarizes commonly used approaches for solubility enhancement. Particle size reduction to nanometer range $(<1 \mu \mathrm{m})$ is an attractive and modern approach for poorly water-soluble drugs and has been explored significantly in last two decades. Particle size reduction could lead to an increase of the surface area and a decrease of the diffusion layer thickness, which could provide an enhanced dissolution rate for drugs as well as an increase in the saturation solubility is also expected by reducing the particle size to less than $1 \mu \mathrm{m}$, as described by Ostwald-Freundlich's equation (Müller and Peters, 1998). The nanocrystal formulations are commonly produced by wet-milling with beads, high- 
Table 1. Commonly used solubility enhancement approaches for drugs

\begin{tabular}{|c|c|c|}
\hline Strategy & Approach & Techniques \\
\hline \multirow[t]{3}{*}{ Physical modification } & Particle size reduction & $\begin{array}{l}\text { Micronization } \\
\text { Nanonization }\end{array}$ \\
\hline & $\begin{array}{l}\text { Modification of the } \\
\text { crystal habit }\end{array}$ & $\begin{array}{c}\text { Polymorphs } \\
\text { Pseudopolymorphs } \\
\text { (including solvates) } \\
\text { Complexation- } \\
\text { Use of cyclodextrins } \\
\text { Solubilization- } \\
\text { Use of surfactants } \\
\end{array}$ \\
\hline & $\begin{array}{l}\text { Drug dispersion } \\
\text { in carrier }\end{array}$ & $\begin{array}{l}\text { Eutectic mixture } \\
\text { Solid dispersion } \\
\text { (non-molecular) } \\
\text { Solid solutions }\end{array}$ \\
\hline Chemical modification & $\begin{array}{c}\text { Structural modification in } \\
\text { drug moiety }\end{array}$ & $\begin{array}{l}\text { Prodrugs } \\
\text { Salt formation }\end{array}$ \\
\hline
\end{tabular}

pressure homogenization, or controlled precipitation (Dahiya, 2017; Shegokar and Muller, 2010). Hydrophilic polymer and/or surfactant are typically used to stabilize nanocrystal suspension. The nanocrystalline drug particles are dispersed into inert carriers after a drying process, such as spray drying or lyophilization. Herein, the solidified nanocrystal formulations can be defined as crystalline solid dispersion (CSD). There have been numerous studies demonstrating the enhanced oral bioavailability of pharmaceuticals and nutraceuticals by nanocrystal technologies (Fakes et al 2009; Hanafy et al 2007; Hecq et al 2006; Jia et al 2002; 2003; Jinno et al 2006; 2008; Kawabata et al 2010). Drug nanocrystals are crystals with a size in the nanometer range, which means they are nanoparticles with a crystalline character.

Nanocrystals are composed of $100 \%$ drug; there is no carrier material as in polymeric nanoparticles. Dispersion of drug nanocrystals in liquid media leads to so called "nanosuspensions" (in contrast to "microsuspensions" or "macrosuspensions"). In general, the dispersed particles need to be stabilized, such as by surfactants or polymeric stabilizers. Dispersion media can be water, aqueous solutions or non-aqueous media (e.g. liquid polyethylene glycol [PEG], oils). Depending on the production technology, processing of drug microcrystals to drug nanoparticles can lead to either crystalline or amorphous product, especially when applying precipitation (Dahiya, 2017). The characteristics of nanoparticles are summarized in the Table 2 .
Table 2. Characteristics of nanocrystals

\begin{tabular}{|c|c|}
\hline No. & Characteristic \\
\hline 1 & Size below $1 \mu \mathrm{m}$ \\
\hline 2 & $100 \%$ drug, no carrier \\
\hline 3 & Crystalline or amorphous structure \\
\hline 4 & Small particle size offers advantage \\
\hline 5 & Enhanced dissolution velocity \\
\hline 6 & Enhanced saturation solubility \\
\hline 7 & Generally needs stabilization \\
\hline
\end{tabular}

\section{Applications of Nanocrystals Oral delivery}

The biological activity/oral bioavailability (BA) of a compound depends on its ability to dissolve and diffuse through the gastrointestinal membranes to the blood. In BCS class II the BA is limited by the dissolution velocity, in class IV there is additionally a transport mechanism reducing the absorption (e.g. p-glycoprotein). In the latter case, absorption might be enhanced by flooding the transporter system with dissolved drug. The faster dissolution of the nanocrystals can be explained by the increase in surface area when moving from micronized to nanonized particles. At the same time, saturation solubility Cs increases below a size of about $1 \mu \mathrm{m}$. The basis for this is the Kelvin equation describing the vapor pressure of a liquid droplet in a gas phase, which corresponds to the dissolution pressure of a solid particle in a liquid. The Cs depends on the size, i.e. the curvature of the particle and the corresponding dissolution pressure. The dissolution pressure increases with increasing curvature, i.e. decreasing particle size. The vapor/dissolution pressure can be 
calculated as a function of size, showing a steep increase below $1 \mu \mathrm{m}$, very pronounced increase below $100 \mathrm{~nm}$ (Müller and Akkar, 2004). The increased dissolution pressure shifts the balance of dissolving/re-crystallizing molecules around a crystal towards the dissolved molecules. A higher kinetic saturation solubility than the thermodynamic equilibration solubility leads to an increased concentration gradient at membranes, subsequently leading to higher penetration or permeation. Both increased Cs and increased surface area enhances the dissolution velocity, as described in the equation by Noyes-Whitney (Noyes and Whitney, 1897). By now, main attention was focused on size and related surface area. It was recently reported that the interfacial reaction resistance is getting the velocity determining parameter for crystals below $1 \mu \mathrm{m}$. Design of nanocrystals with faster interfacial reaction can further enhance the dissolution velocity (Crisp et al 2007). In addition, nanomaterials possess improved adhesiveness to biological membranes. In many studies, these principles of actions for nanocrystals were employed. Administration of atovaquone (an antibiotic used in treating opportunistic Pneumocystis carinii infections) as a nanosuspension resulted in a 2.5 -fold increase in oral bioavailability as compared to the commercial product Wellvone, which contains the micronized drug (Schöler et al 2001). The enhancement in oral bioavailability can be attributed to the adhesiveness of the drug nanosuspension, increased surface area (due to reduction in particle size by 10 to 50 fold) and saturation solubility. Danazol (gonadotropin inhibitor) showed a drastic improvement in bioavailability to $82.3 \%$ when administered as a nanosuspension as compared to marketed danazol macrosuspension (Danocrine) which showed poor bioavailability of 5.2\% (Liversidge and Cundy, 1995). Oral administration of amphotericin $\mathrm{B}$ as a nanosuspension produced a substantial improvement in its oral absorption in comparison to orally administered conventional commercial formulations such as Fungizone, AmBisome and micrometer amphotericin B (Kayser, 2001). Apart from improving oral absorption, nanosuspensions offer improved dose proportionality, reduced fed/fasted state variability and reduced intersubject variability. Another examples for oral nanocrystals is the anthelmintic drug albendazole, showing an about five times higher saturation solubility compared to the raw material (Ravichandran, 2010). In another study, 1,3-dicyclohexylurea (DCU) was formulated as a nanosuspension to lower systemic blood pressure. The test model was hypertensive rats dosed intraperitoneally. The DCU nanosuspension administered orally twice daily yielded plasma exposures an order of magnitude greater than raw material (Ghosh et al 2008).

Nanosuspensions exhibit a quick onset of action for drugs that are completely but slowly absorbed like naproxen (NSAID) for which dosage form with fast onset of action would be highly desirable. Naproxen nanosuspension showed a reduction in $\mathrm{t}_{\max }$ by approximately $50 \%$ to achieve $\mathrm{C}_{\max }$ as compared to the suspension (Naprosyn) and the tablet (Anaprox), besides an increase in bioavailability (MeriskoLiversidge et al 2003). In some cases, quick onset and the associated high $\mathrm{C}_{\max }$ is not desired. In this case, the nanocrystals need to be incorporated into prolonged release dosage forms, e.g. pellets (Möschwitzer and Müller, 2006). This is sensible approach for delivery of BCS class IV drugs, addition of inhibitors of the efflux transporters. Nanocrystals can be easily transformed to solid oral dosage forms. The aqueous nanosuspension can be used as granulation fluid in tablet production, or as wetting fluid for the mass in pelletization. Aqueous nanosuspensions can be transferred to powders by spray-drying or lyophilization. Important is that the dry product redisperses well in water, with little increase in the size (i.e. little aggregates). The powder can be compressed into tablets. The enhanced dissolution behaviour for a rutin tablet made from nanocrystals compared to a marketed product was found and even larger differences to marketed products were obtained for the nutraceuticals coenzyme Q10 and hesperidin (Mauludin, 2008). Table 3 lists the marketed nanocrystal products.

\section{Parenteral delivery}

Parenteral administration of poorly soluble drugs requires often the use of a solubilization technology, at least when doses have to be administered being not soluble in typical injection (1-10 ml) or infusion volumes (e.g. 100 $\mathrm{ml}$ ). Approaches being used are based on solubilization by surfactants (e.g. Cremophor ${ }^{\circledR}$ EL in Taxol®), solvent mixtures (e.g. ethanolwater) or cyclodextrins inclusion complexes. However, many drugs of today are so poorly soluble that these approaches do not work, or 
Table 3. Marketed formulations based on nanocrystal technology

\begin{tabular}{|c|c|c|c|}
\hline Trade name & Drug & Indication & Company \\
\hline Rapamune $^{\circledR}$ & Rapamycin & Immunosuppressive & Wyeth \\
\hline Emend $^{\circledR}$ & Aprepitant & Antiemetic & Merck \\
\hline Tricor $^{\circledR}$ & Fenofibrate & Hypercholestrerolemia & Abbott \\
\hline Triglide $^{\circledR}$ & Fenofibrate & Hypercholestrerolemia & SkyePharma \\
\hline Megace ES $^{\circledR}$ & Megestrol acetate & Antianorexic & $\begin{array}{c}\text { Par Pharmaceuticals } \\
\text { Company }\end{array}$ \\
\hline
\end{tabular}

these approaches work, but are associated with undesired side effects. Examples are anaphylactic reaction in products with Cremophor® EL, (Irizarry et al 2009) or nephrotoxicity when used cyclodextrins intravenously (Rabinow et al 2007). Nano delivery systems are of increasing interest for not only oral but also parenteral administration, e.g. microemulsions but also nanosuspensions as described for benzimidazoles (Chow et al 2010). Nanosuspensions are a smart approach to solve both problems i.e. insufficient solubility and side effects. They can be produced using welltolerated stabilizers, and can be injected in concentrations up to $10 \%(w / w)$ without obvious problems (unpublished data from animal studies). The product Sporanox® IV (itraconazole, Janssen Pharmaceutical Products, L.P.) exhibited significant acute toxicity above 10 $\mathrm{mg} / \mathrm{kg}$ and an $\mathrm{LD}_{50}$ value lower than $40 \mathrm{mg} / \mathrm{kg}$ when administered as a bolus in the caudal vein of rats. Itraconazole nanosuspension could be administered up to $320 \mathrm{mg} / \mathrm{kg}$ without animal mortality. Itraconazole nanosuspensions were developed and intensively investigated by Baxter Healthcare (Pandey, 2010; Rabinow et al 2007). The maximum tolerable dose of paclitaxel nanosuspension was found to be three times higher than the currently marketed Taxol (Böhm, 1999). To optimize the stability of nanocrystal suspensions can be a rather complex process as shown for specifically paclitaxel (Deng et al 2010). There is a complex process of adsorption and desorption of polymeric stabilizer on the nanocrystals surface, depending on polymer concentration and temperature. Similarly to paclitaxel nanosuspension, the nanosuspensions of etoposide and camptothecin revealed an improvement in the tolerance level of the drug compared to the marketed preparations (Merisko-Liversidge et al 2003). Clofazimine (antileprotic drug) nanosuspension showed an improvement in stability and efficacy over the liposomal clofazimine (Peters et al 2000).
A nimodipine i.v. nanosuspension was developed to have an injectable form better tolerated than the commercially available ethanol solution. Irritability studies in rats showed less local irritation and less phlebitis risks (Xiong et al 2008). It has to be taken into account, that i.v. injected nanocrystals exhibit a different pharmacokinetics compared to an injected solution, when the nanocrystals are larger than $100 \mathrm{~nm}$. The nanocrystals do not dissolve fast enough and are sequestered by the mononuclear phagocytic system (MPS) cells. They accumulate mainly in the Kupffer cells of the liver like other colloidal drug carriers, e.g. shown for Paclitaxel nanosuspensions (Böhm, 1999). That means a generic product to Taxol® cannot be produced using such large-sized nanocrystals. As positive effect, this accumulation in the liver leads to prolonged plasma levels. The macrophages act as depot and drug is being released over time to the blood. As potential side effect, local high drug concentrations can cause toxicity to liver. One needs to find a balance by selecting the right injected dose. Asulacrine nanosuspension was developed for i.v. treatment of breast cancer. It showed a different pharmacokinetics to the injected solution, and enrichment in liver, lung and kidney (Ganta et al 2009). Injected nanocrystals can also be used to target drugs to various vital organs (Shegokar, 2010). Accumulation in the liver is "natural" or "passive" targeting, it happens automatically. To achieve macrophage targeting in certain diseases like HIV/AIDS, tuberculosis (TB) the surface properties of nanosuspensions could be modulated in a controlled way to alter the plasma protein adsorption pattern. This directs particles away from the liver to the spleen. The uptake pattern within the different cells of the MPS can be changed to treat most efficiently a disease.

To direct nanocrystals to other sides in the body (active targeting), identical to stealth liposomes, the surface of the nanocrystals needs to be masked to avoid opsonin adsorption and 
recognition by the MPS (Shegokar and Singh, 2009). In addition, the surface coating needs to have attached a targeting moiety, e.g. antibody or targeting protein (Shegokar et al 2010). Literature is successful to target darlagin loaded onto polymeric nanoparticles to the brain. The particles were surface-modified by Tween 80 adsorption (Kreuter et al 1997). This coating leads to preferential adsorption of Apolipoprotein E after i.v. injection. The Apo E mediated the targeting to the endothelial cells of the blood-brain barrier (Lück, 1997). This principle was transferred to atovaquone nanocrystals. The parasites in toxoplasmosis could be eradicated in the brain (Schöler et al 2001). The challenge in targeting to other sides than the liver and spleen is to enrich a sufficiently high percentage of the injected dose in the target area. There is still a competition in uptake by the MP Sand the target area. In brain delivery, it is estimated that not more than about $1 \%$ of the injected dose reaches the brain. This is the present hurdle for targeting with i.v. nanocrystals. To mimic injected solutions, the nanocrystals need to dissolve very fast, i.e. they should be $<100 \mathrm{~nm}$. The difference in organ distribution as a function of nanocrystals size was shown for oridonin nanosuspensions with a size of $103 \mathrm{~nm}$ versus $897 \mathrm{~nm}$. The small nanocrystals exhibited a pharmacokinetics and organ distribution similar to the injected solution, the large nanocrystals accumulated in the RES organs. Of course, it does not depend on the size only, but also on the compound specific dissolution pressure. For such formulations, the second generation nanocrystal technology is required providing such small sizes. Moreover, nanosuspensions can also be administered via other parenteral routes, e.g. intraarticular and intraperitoneal. Replacing an intraperitoneally injected microsuspension by a nanosuspension could avoid irritation of the peritoneum (unpublished data). Literature is enriched with preclinical studies reports for subcutaneous application of nanocrystal formulations (Wolf et al 1999). However, by now these routes are not intensively studied, almost nothing is published. Nanocrystals are also a formulation principle in early drug discovery and screening. In the assessment of pharmacodynamic responses in early drug screening, very often one needs "tool compounds". These tool compounds are often poorly soluble. The poorly soluble 1,3dicyclohexylurea (DCU) reduces blood pressure, and was formulated as nanosuspension for intravenous bolus and infusion dosing (Wahlstrom et al 2007).

\section{Dermal and mucosal delivery}

Nanocrystals exhibit the properties like increased penetration into a membrane, enhanced permeation and bioadhesiveness. These principles were exploited to the gastrointestinal wall for the oral products. The injectability and fast dissolution was exploited for intravenous formulation developments. However, for many years no attention was given to exploit adhesion, fast dissolution and increased penetration for dermal and mucosal application. This changed when the poorly soluble antioxidants rutin, apigenin and hesperidin were formulated as nanosuspension for application in skin-protective, anti-aging cosmetic products (Al Shaal et al 2010a; Mauludin et al 2009). The nanocrystals are simply admixed to the water phase of dermal creams and $\mathrm{o} / \mathrm{w}$ lotions. The first products with rutin appeared on the market in March 2007, series Juvedical, age-decoder face cream and fluid. It was followed by Platinum Rare in 2009, containing hesperidin. These products contain nanosized crystals, but are not a nano product according to the new European regulations for cosmetics, as the size of the nanocrystals is above $100 \mathrm{~nm}$, and the particles are not biopersistent, they are biodegradable. Rutin nanocrystals formulations were compared to a cream with a water soluble rutin derivative in vivo. The concentration of dissolved active in the water phase was $1 / 500$ in the nanocrystals formulation, but the Sun Protection Factor (SPF) was still higher for the nanocrystal formulation as compared to water soluble derivative (Petersen, 2006). The underlying mechanism of action is that the nanocrystals increase the solubility of the poorly soluble active in the water phase, this leads to an increased concentration gradient between formulation and skin, thus increased penetration compared to micronized powder. The original molecule rutin is more lipophilic, therefore penetrates better than the hydrophilic derivative. In addition, the original molecule might have more activity in the cell than the derivative. Active penetrated from the water phase into the skin is rapidly replaced by fast dissolving active from the nanocrystals, they act as depot in the water phase. The same principle can be applied to pharmaceutical dermal formulations. Diclofenac sodium nanosuspension for transdermal delivery 
showed increased permeability flux of drug across the skin by up to 3.8 fold compared to the control when tested in Yucatan micropig (YMP) skin model (Piao et al 2008). Similarly antioxidant activity of hesperetin as nanocrystals was significantly increased when tested in vitro by radio scavenging method (Al Shaal et al 2010b) and can be used as effective adjuvant in skin care or dermatological preparation.

Basically the same nanosuspensions can be applied to mucosal surfaces, either as nanosuspension spray, or lotions. The adhesive effect due to the nanosize can further be enhanced by the use of positively charged polymers as stabilizers for the drug nanocrystals. The opposite charge leads to an increased affinity of the drug nanocrystals to the negatively charged cells (unpublished data). This principle was previously shown by producing antiseptic lipid nanoparticle sprays, using cetylpyridinium chloride as cationic surfactant and antiseptic at the same time. Ultrafine lidocaine base nanocrystals were prepared as prolonged release formulation for dermal use using the combination technology (Shegokar et al 2010). Vaginal and rectal administration can treat local STD (sexually transmitted diseases) by evenly spreading of drug in the local area.

\section{Ocular delivery}

Nanosuspensions have not been yet exploited for this route of drug administration. The general problem is that solutions are relatively fast cleared from the eye, adhesive nanoparticulate suspensions can show prolonged release due to their adhesion properties. Polymeric (Eudragit RS 100 and Eudragit RL 100) nanoparticulate suspensions of flurbiprofen and ibuprofen (Bucolo et al 2002) revealed superior in vivo performance over the existing marketed formulations and could sustain drug release for $24 \mathrm{~h}$. This proves the principle. Another example is acyclovir loaded Eudragit RS polymeric nanoparticle suspensions (Dandagi et al 2009). Drug nanosuspensions can also be used for drugs that exhibit poor solubility in lachrymal fluids providing advantages of prolonged residence time in a cul-de-sac. Also, there are few studies which investigated NSAIDs in the form of nanocrystals for ophthalmic application (Araújo et al 2009). An increased rate and extent of drug absorption and intensity of drug action was reported for ocular nanosuspensions of the hydrocortisone, prednisolone and dexa- methasone (Kassem et al 2007). In contrast to the polymeric nanoparticles, nanosuspensions have a clear regulatory advantage. The particles are drained via the lipophilic channels to the nose; from nose to the pharynx. That means, the materials used in formulation need to be approved for occular administration. As many polymers are not approved by official authorities. As nanosuspensions do not contain any matrix material, and are purely composed of drug and comparatively small amount of stabilizer. Many stabilizers suitable for stabilization of nanosuspensions are listed in GRAS catalogue (USFDA).

\section{Pulmonary delivery}

As an alternative to dry powders for inhalation, nanosuspensions can be used in case of poorly soluble drugs. Application can simply be performed by placing aqueous nanosuspensions in an aqueous nebulizer, e.g. Pari Boy, or use portable nebulizers on the market. The nebulizer generates an aerosol, with a droplet size suitable for pulmonary administration, e.g. 1-5 $\mu \mathrm{m}$ droplets. The nanocrystals are contained inside these droplets. The nanocrystals cannot be inhaled as a powder. First of all, the nanocrystals are highly adhesive with a tendency to agglomerate, and in addition particles below $0.5-1 \mu \mathrm{m}$ are being exhaled. The advantage of nanocrystals is that they show an increased dissolution velocity compared to micron-sized crystals. When the aerosol droplets deposit in the lung, as fine particles they should spread more evenly on the lung surface, especially when stabilized with surfactants with good spreadability. Budesonide (corticosteroid) nanosuspensions for pulmonary delivery have been successfully formulated (Müller et al 2001).

\section{Targeted delivery}

The need to target drugs to specific sites by means of nanoparticles is increasing day by day as a result of therapeutic and economic factors. Nanosuspensions can be used for targeted delivery as their surface properties and in vivo behavior can easily be altered. Their versatility and ease of scale-up enable the development of commercially viable nanosuspensions. The engineering of stealth nanosuspensions (analogous to stealth liposomes) by using various surface coatings for active or passive targeting is the future of targeted drug delivery systems. Targeting of Cryptosporidium parvum (cryptosporidiosis) by using surface-modified 
mucoadhesive nanosuspensions of bupravaquone is reported in literature (Kayser, 2001). A superior targeting was achieved for mucoadhesive bupravaquone nanosuspensions, because of their prolonged residence at infection site. They showed a 10 -fold reduction in the infectivity score of Cryptosporidium parvum as compared to the bupravaquone nanosuspensions without mucoadhesive polymers. Similarly, pulmonary aspergillosis can easily be targeted by using amphotericin B nanosuspensions instead of using stealth liposomes (Kohno et al 1997). Targeting of HIV viral reservoirs was successfully achieved by using bare and coated nevirapine nanosuspensions (Shegokar et al 2009). In addition, nanosuspensions can be used as supportive treatment in various disease conditions like cancer (Shegokar et al 2010), TB and HIV/AIDS. Important for intravenous targeting is the adjustment of the surface properties of the nanocrystals. The surface properties determine the qualitative and quantitative composition of the adsorption patterns of blood proteins (Blunk et al 1993). These adsorbed proteins determine subsequently the fate of the injected particles in the body. Fate means recognition by the MPS system and primarily accumulation in liver and spleen, circulation in the blood as stealth particles or enrichment at other sites, e.g. brain or bone marrow. The surface properties can be adjusted such a way, that the particles even adsorb automatically the blood proteins responsible for enrichment at the desired target site.

\section{Nanocrystals in nutrition}

There is an increasing consciousness about nutritional health and an increasing demand to complement the daily nutrition by additives or nutraceuticals. From the philosophy for a healthy population, nutrition plays a very important role. The nutraceutical market is growing, and there are many nutraceutical

\section{REFERENCES}

Al Shaal L, Shegokar R, Müller RH. Apigenin smart Crystals for novel UV skin protection formulations. Eighth European Workshop on Particulate Systems, 2010a, Abstract No. 22.

Al Shaal L, Shegokar R, Müller RH. Novel UV skin protective antioxidant nanocrystals. First Combined Annual Meeting of the International Pharmaceutical Federation, PSWC and AAPS, 2010b, Abstract No. 2221.

Araújo J, Gonzalez E, Egea MA, Garcia ML, Souto EB. compounds, e.g. antioxidants, which are poorly soluble. Presently most popular molecules is Coenzyme Q10 capsules, but Q10 has a low oral bioavailability. There are products on the market, claiming to contain "nano Q10" being $100 \%$ bioavailable (e.g. containing surfactants for solubilization), requiring only one tenth of the regular dose in normal products. Nanocrystals are also a suitable formulation technology for poorly soluble nutraceuticals like Coenzyme Q10, rutin, hesperidin, apigenin etc.

\section{CONCLUSION}

The small particle sizes have a direct impact on the dissolution rate and bioavailability of poorly soluble drugs after oral, topical, and parenteral administration which in turn implies improved in vivo performance. Despite huge potential and numerous applications, there are many technical limitations related to nanocrystal technology which mainly includes its applicability to only BCS class II drugs, difficulty in characterization, possible nanotoxicity and high production cost. More research needs to be performed, however, to solve the technical challenges of the different technologies in order to achieve improved particle size reduction effectiveness and better formulations for new, problematic compounds. To date, some nanocrystal delivery systems are under pipeline for clinical studies and many more are under academic investigation, but only few made it to the market. This may be due to missing nanotoxicity and cytotoxicity data, lack of regulatory accepted status of the excipients, lack of large scale production lines which can be validated and acceptable by the regulatory authorities. In future, more drugs will be poorly soluble requiring smart formulation technologies and delivery for enhanced bioavailability. Further, by modifying the nanocrystal surface, it is possible to achieve a prolonged or targeted release. This could be an important part of the nanocrystal research which might prove a breakthrough in drug delivery science.

Nanomedicines for ocular NSAIDs: safety on drug delivery. Nanomedicine 2009;5(4):394-401.

Blunk T, Hochstrasser DF, Sanchez JC, Muller BW, Muller RH. Colloidal carriers for intravenous drug targeting: plasma protein adsorption patterns on surface-modified latex particles evaluated by two-dimensional polyacrylamide gel electrophoresis. Electrophoresis 1993; 14(12):1382-7.

Böhm BHL. Herstellung und Charakterisierung von Nanosuspensionen als neue Arzneiform für Arzneistoffe 
mit geringer Bioverfügbarkeit. Ph.D. Thesis, Freie Universität Berlin, Germany, 1999.

Bucolo C, Maltese A, Puglisi G. Enhanced ocular antiinflammatory activity of ibuprofen carried by an Eudragit RS100 nanoparticle suspension. Ophthal. Res. 2002; 34(5):319-23.

Chow DSL, Gupta P, Qi Y, Liang D, Shah J, Wisniecki P. Parenteral and oral formulations of benzimidazoles, 2010, WO/2010/011289.

Crisp MT, Tucker CJ, Rogers TL, Williams RO 3rd, Johnston KP. Turbidimetric measurement and prediction of dissolution rates of poorly soluble drug nanocrystals. $J$. Control. Release 2007;117(3):351-9. [DOI: 10.1016/j.jcon rel.2006.11.011]

Dahiya S. Drug nanonization: an overview of industrially feasible top-down technologies for nanocrystal production. Bull. Pharm. Res. 2017;7(2):144. [DOI: 10.212 76/bpr.2017.7.2.2]

Dandagi P, Kerur S, Mastiholimath V, Gadad A, Kulkarni A. Polymeric ocular nanosuspension for controlled release of acyclovir: in vitro release and ocular distribution. Iran. J. Pharm. Res. 2009;8(2):79-86.

Deng J, Huang L, Liu F. Understanding the structure and stability of paclitaxel nanocrystals. Int. J. Pharm. 2010; 390(2):242-9. [DOI: 10.1016/j.ijpharm.2010.02.013]

Fakes MG1, Vakkalagadda BJ, Qian F, Desikan S, Gandhi RB, Lai C, Hsieh A, Franchini MK, Toale H, Brown J. Enhancement of oral bioavailability of an HIV-attachment inhibitor by nanosizing and amorphous formulation approaches. Int. J. Pharm. 2009;370(1-2):167-74. [DOI: 10.1016/j.ijpharm.2008.11.018]

Ganta S, Paxton JW, Baguley BC, Garg S. Formulation and pharmacokinetic evaluation of an asulacrine nanocrystalline suspension for intravenous delivery. Int. J. Pharm. 2009;367(1-2):179-86. [DOI: 10.1016/j.ijpharm. 2008.09.022]

Ghosh S, Chiang PC, Wahlstrom JL, Fujiwara H, Selbo JG, Roberds SL. Oral delivery of 1,3-dicyclohexylurea nanosuspension enhances exposure and lowers blood pressure in hypertensive rats. Basic Clin. Pharmacol. Toxicol. 2008;102(5):453-8. [DOI: 10.1111/j.1742-7843. 2008.00213.x]

Hanafy A, Spahn-Langguth H, Vergnault G, Grenier P, Tubic Grozdanis M, Lenhardt T, Langguth P. Pharmacokinetic evaluation of oral fenofibrate nanosuspensions and SLN in comparison to conventional suspensions of micronized drug. Adv. Drug Del. Rev. 2007;59(6):419-26. [DOI: 10.101 6/j.addr.2007.04.005]

Hecq J, Deleers M, Fanara D, Vranckx H, Boulanger P, Le Lamer S, Amighi K. Preparation and in vitro/in vivo evaluation of nano-sized crystals for dissolution rate enhancement of ucb-35440-3, a highly dosed poorly water soluble weak base. Eur. J. Pharm. Biopharm. 2006; 64(3):360-8. [DOI: 10.1016/j.ejpb.2006.05.008]

Irizarry LD, Thanh HL, McKoy JM, Samaras AT, Fisher MJ, Carias EE, Raisch DW, Calhoun E, Bennett CL. Cremophor EL-containing paclitaxel induced anaphylaxis: a call to action. Commun. Oncol. 2009;6(3):132-4.

Jia L, Wong H, Cerna C, Weitman SD. Effect of nanonization on absorption of 301029: ex vivo and in vivo pharmacokinetic correlations determined by liquid chromatography/mass spectrometry. Pharm. Res. 2002; 19(8):1091-6. [DOI: 10.1023/A:1019829622088]

Jia L, Wong H, Wang Y, Garza M, Weitman SD. Carbendazim: disposition, cellular permeability, metabolite identification, and pharmacokinetic comparison with its nanoparticle. Pharm. Res. 2003;92(1):161-72. [DOI: 10.10 02/jps.10272]

Jinno J, Kamada N, Miyake M, Yamada K, Mukai T, Odomi M, Toguchi H, Liversidge GG, Higaki K, Kimura T. Effect of particle size reduction on dissolution and oral absorption of a poorly water-soluble drug, cilostazol, in beagle dogs. J. Control. Release 2006;111(1-2):56-64. [DOI: 10.1016/ j.jconrel.2005.11.013]

Jinno J, Kamada N, Miyake M, Yamada K, Mukai T, Odomi M, Toguchi H, Liversidge GG, Higaki K, Kimura T. In vitro-in vivo correlation for wet milled tablet of poorly watersoluble cilostazol. J. Control. Release 2008;130(1):29-37. [DOI: 10.1016/j.jconrel.2008.05.013]

Junghanns JA, Muller RH. Nanocrystal technology, drug delivery and clinical applications. Int. J. Nanomed. 2008; 3(3):295-309. [DOI: 10.2147/IJN.S595]

Kassem MA1, Abdel Rahman AA, Ghorab MM, Ahmed MB, Khalil RM. Nanosuspension as an ophthalmic delivery system for certain glucocorticoid drugs. Int. J. Pharm. 2007;340(1-2):126-33. [DOI: 10.1016/j.ijpharm.2007.03. 011]

Kawabata Y, Yamamoto K, Debari K, Onoue S, Yamada S. Novel crystalline solid dispersion of tranilast with high photostability and improved oral bioavailability. Eur. J. Pharm. Sci. 2010;39(4):256-62. [DOI: 10.1016/j.ejps.200 9.12.009]

Kayser 0. A new approach for targeting to cryptosporidium parvum using mucoadhesive nanosuspensions: Research and applications. Int. J. Pharm. 2001;214(1-2):83-5. [DOI: 10.1016/S0378-5173(00)00640-2]

Kohno S, Otsubo T, Tanaka E, Maruyama K, Hara K. Amphotericin B encapsulated in polyethylene glycolimmunoliposomes for infectious diseases. Adv. Drug Deliv. Rev. 1997;24(2-3):325-9. [DOI: 10.1016/S0169-409X(96) 00474-7]

Kreuter J, Petrov VE, Kharkevich DA, Alyautdin ab RN. Influence of the type of surfactant on the analgesic effects induced by the peptide dalargin after its delivery across the blood-brain barrier using surfactant-coated nanoparticles. J. Control. Release 1997;49(1):81-7. [DOI: 10.1016/S0168-3659(97)00061-8]

Liversidge GG, Cundy KC. Particle size reduction for improvement of oral bioavailability of hydrophobic drugs: I. Absolute oral bioavailability of nanocrystalline danazol in beagle dogs. Int. J. Pharm. 1995;125(1):91-7. [DOI: 10.1 016/0378-5173(95)00122-Y]

Lück M. Plasmaproteinadsorption als ein möglicher Schlüsselfaktor für eine kontrollierte Arzneistoffapp liktion mit partikulären Trägern. Ph.D. Thesis, Freie Universität Berlin, 1997.

Mauludin R. Nanosuspensions of poorly soluble drugs for oral administration. Ph.D. Thesis, Freie Universität Berlin, Germany, 2008.

Mauludin R, Müller RH, Keck CM. Development of an oral rutin nanocrystal formulation. Int. J. Pharm. 2009;370(12):202-9. [DOI: 10.1016/j.ijpharm.2008.11.029]

Merisko-Liversidge E, Liversidge GG, Cooper ER. Nanosizing: a formulation approach for poorly-watersoluble compounds. Eur. J. Pharm. Sci. 2003;18(2):113-20. [DOI: 10.1016/S0928-0987(02)00251-8]

Moschwitzer J, Muller RH. Spray coated pellets as carrier system for mucoadhesive drug nanocrystals. Eur. J. Pharm. Biopharm. 2006;62(3):282-7. [DOI: 10.1016/j.ejp b.2005.09.005]

Müller RH, Jacobs C, Kayser O. Nanosuspensions as particulate drug formulations in therapy. Rationale for 
development and what we can expect for the future. $A d v$. Drug Deliv. Rev. 2001;47(1):3-19.

Muller RH, Akkar A. Drug nanocrystals of poorly soluble drugs. In: Nalwa HS (ed) Encyclopedia of nanoscience and nanotechnology, American Scientific Publishers: California, USA, 2004; 627-38.

Muller RH, Peters K. Nanosuspensions for the formulation of poorly soluble drugs: I. preparation by a size-reduction technique. Int. J. Pharm. 1998;160(2):229-37. [DOI: 10.10 16/S0378-5173(97)00311-6]

Noyes AA, Whitney WR. The rate of solution of solid substances in their own solutions. J. Am. Chem. Soc. 1897;19(12):930-4. [DOI: 10.1021/ja02086a003]

Pandey S. Itraconazole nanosuspension meant for oral use: Development of formulation, characterization and in vitro comparison with some marketed formulations. Der Pharm. Lett. 2010;2(1):162-71.

Peters K, Leitzke S, Diederichs JE, Borner K, Hahn H, Müller $\mathrm{RH}$, Ehlers S. Preparation of a clofazimine nanosuspension for intravenous use and evaluation of its therapeutic efficacy in murine Mycobacterium avium infection. J. Antimicrob. Chemother. 2000;45(1):77-83.

Petersen R. Nanocrystals for use in topical cosmetic formulations and method of production thereof, 2006, US Patent 60/8866233.

Piao H, Kamiya N, Hirata A, Fujii T, Goto M. A novel solid-inoil nanosuspension for transdermal delivery of diclofenac sodium. Pharm. Res. 2008;25(4):896-901. [DOI: 10.1007/ s11095-007-9445-7]

Rabinow B, Kipp J, Papadopoulos P, Wong J, Glosson J, Gass J, Sun CS, Wielgos T, White R, Cook C, Barker K, Wood K. Itraconazole IV nanosuspension enhances efficacy through altered pharmacokinetics in the rat. Int. J. Pharm. 2007;339(1-2):251-60. [DOI: 10.1016/j.ijpharm.2007.02. 030]

Ravichandran R. In vivo pharmacokinetic studies of albendazole nanoparticulate oral formulations for improved bioavailability. Int. J. Green Nanotechnol. Biomed. 2010;2(1):B46-53. [DOI: 10.1080/1943085x.201 0.488200]
Schöler N, Krause K, Kayser O, Müller RH, Borner K, Hahn H, Liesenfeld 0 . Atovaquone nanosuspensions show excellent therapeutic effect in a new murine model of reactivated toxoplasmosis. Antimicrob. Agents Chemother. 2001;45(6):1771-9. [DOI: 10.1128/AAC.45.6.1771-1779. 2001]

Shegokar R, Singh KK. Antiretroviral nanosuspensions for latent HIV reservoir targeting. 36th Annual Meeting and Exposition of the Controlled Release Society, Copenhagen, Denmark, 2009, Abstract No. 588.

Shegokar R, Singh KK, Müller RH. Targeting to Latent HIV reservoirs: production optimization and evaluation of nevirapine nanosuspensions, 69th International Congress of FIP. Istanbul, Turkey, 2009, Abstract No. 451.

Shegokar R. Development and evaluation of novel drug delivery systems for an anti-HIV Drug. Ph.D. Thesis, S.N.D.T. Women's University, Mumbai, India, 2010.

Shegokar R, Muller RH. Nanocrystals: industrially feasible multifunctional formulation technology for poorly soluble actives. Int. J. Pharm. 2010;399(1-2):129-39. [DOI: 10.1016/j.ijpharm.2010.07.044]

Shegokar R, Al Shaal L, Müller RH. Local anaesthetic nanocrystal as prolonged release formulation. First Combined Annual Meeting of the International Pharmaceutical Federation, PSWC and AAPS New Orleans, Louisiana, USA, 2010, Abstract no. 2203.

Wahlstorm JL, Chiang P-C, Ghosh S, Warren CJ, Wene SP, Albin LA, Smith ME, Roberds SL. Pharmacokinetic evaluation of a 1,3-dicyclohexylurea nanosuspension formulation to support early efficacy assessment. Nanoscale Res. Lett. 2007;2(6):291-6.

Wolf GL, Shore MT, Bessin G, Mclntire GL, Bacon ER, Illig KJ. Lymph node extraction of radiopaque nanoparticulates in the rabbit as measured in vivo with CT. Acad. Radiol. 1999;6(1):55-60. [DOI: 10.1016/S1076-6332(99)800622]

Xiong R, Lu W, Li J, Wang P, Xu R, Chen T. Preparation and characterization of intravenously injectable nimodipine nanosuspension. Int. J. Pharm. 2008;350(1-2):338-43. [DOI: 10.1016/j.ijpharm.2007.08.036] 